\title{
Editorial
}

\section{Multispectral, Hyperspectral, and Polarimetric Imaging Technology}

\author{
Yongqiang Zhao, ${ }^{1}$ Jonathan Cheung-Wai Chan, ${ }^{2}$ Seong G. Kong, \\ Yanli Qiao, ${ }^{4}$ Chao-Cheng Wu, ${ }^{5}$ and Chein-I. Chang ${ }^{6}$ \\ ${ }^{1}$ School of Automation, Northwestern Polytechnical University, Xian, Shanxi 710072, China \\ ${ }^{2}$ Department of Electronics and Informatics (ETRO), Vrije Universiteit Brussel (VUB), Pleinlaan 2, 1050 Brussels, Belgium \\ ${ }^{3}$ Department of Computer Engineering, Sejong University, Seoul 143-747, Republic of Korea \\ ${ }^{4}$ Anhui Institute of Optics and Fine Mechanics, Chinese Academy of Sciences, Hefei, Anhui 230031, China \\ ${ }^{5}$ Department of Electrical Engineering, National Taipei University of Technology, Taipei 10608, Taiwan \\ ${ }^{6}$ Remote Sensing Signal and Image Processing Laboratory (RSSIPL), University of Maryland Baltimore County (UMBC), \\ Baltimore, MD 21201, USA \\ Correspondence should be addressed to Yongqiang Zhao; zhaoyq@nwpu.edu.cn
}

Received 2 July 2015; Accepted 7 July 2015

Copyright ( 2016 Yongqiang Zhao et al. This is an open access article distributed under the Creative Commons Attribution License, which permits unrestricted use, distribution, and reproduction in any medium, provided the original work is properly cited.

This special issue presents papers that describe research in multispectral, hyperspectral, and polarimetric image processing and applications. The subject matter covered ranges from a review of multiband polarization imaging to a presentation of hyperspectral image reconstruction.

Additionally to intensity and spectrum, polarization is a powerful light parameter that contains important information about the diffusion and reflection of light. Spectral imaging sensor is the one that captures image data at specific frequencies across the electromagnetic spectrum, which includes multispectral imager and hyperspectral imager. Polarimetric imaging is the technology on quantitative measurements of this parameter by imaging sensors. Multispectral, hyperspectral, and polarimetric imaging has demonstrated strong potential to retrieve critical information from the scene of interest in challenging imaging conditions. With the advance of imaging sensor's resolution and sensitivity, imaging spectrometry and polarimetry have emerged over the past several decades as a powerful tool to enhance the information availability in computer vision, remote sensing, and biomedicine. Applications of multispectral, hyperspectral, and polarimetric imaging include specular highlight removal, information enhancement in the shadow, penetration through scattering media, investigation of the materials as well as biological tissues, and characterization of atmospheric aerosols and cloud particles.

This special issue contains 10 papers describing research in multispectral, hyperspectral, and polarimetric imaging and applications. The paper by Y. Zhao et al. is a review of multiband polarization imaging. This paper reviews earlier attempts and recent advances in multiband polarization imaging techniques and their applications in various fields such as atmospheric observation, object detection and classification, medical diagnostics, and surveillance. It also introduces the future development trend of multiband polarization imaging techniques. In another paper, T. Liang et al. introduce the airborne polarimetric remote sensing system designed by Anhui Institute of Optics and Fine Mechanics, Chinese Academy of Science. By using the airborne remote sensing images which were acquired on the north coast areas of China during the haze weather, they proved the potential of this system for atmospheric correction. C. Xing et al. describe a hyperspectral image classification method based on deep learning; they utilize a stacked denoise autoencoder (SDAE) method to pretrain the network. Hyperion, AVIRIS, and ROSIS hyperspectral data is used to test the classification performance. H. Ogihara et al. introduce a gastric cancer diagnostic support system using hyperspectral camera. In 
this system, the problem of selecting the optimum wavelength and optimizing the cutoff value is very important, and authors solved it by pattern recognition. $H$. Steiner et al. design an active multispectral SWIR camera system that acquires fourband multispectral image stacks in real-time; they also set up a database containing RGB and multispectral SWIR face images for open access. W. Bao and X. Yao represent a remote sensing images segmentation method by combining the spectrum, shape, and texture features based on graph theory. Z. Miao and W. Shi introduce a new hyperspectral image classification method based on spectral-spatial features. Z. Miao et al. propose the method of accurate road centerline extraction from the classified image. S. Zhao et al. analyze the land use transformation rule in Beijing-Tianjin-Tangshan Region, an important industrial base in China, by using remote sensing and GIS technology. They acquire the land use distribution status at 2000, 2005, and 2010 in Beijing-TianjinTangshan Region using remote sensing images, field survey data, images in Google Earth, and visual interpretation methods. Then, the land use transformation rules from 2000 to 2010 are achieved using GIS (geographic information system) technology and land use distribution status. L. Liu et al. propose an undersampled hyperspectral image reconstruction based on surfacelet transform. They use surfacelet to sparsify the hyperspectral images. Besides, a GramSchmidt orthogonalization is used in CS random encoding matrix, two-dimensional and three-dimensional orthogonal compressed sensing random encoding, and a patch-based compressed sensing encoding scheme is designed.

Whether your interest is in the multispectral, hyperspectral, and polarimetric imaging systems, the image processing methods, or the applications, we hope you enjoy this special issue.

\section{Acknowledgment}

We extend our appreciation to all the authors and reviewers who have worked hard to produce a high-quality issue covering a wide range of topics that represent some of the interesting work being conducted currently in the field of multispectral, hyperspectral, and polarimetric imaging and applications.

Yongqiang Zhao
Jonathan Cheung-Wai Chan
Seong G. Kong
Yanli Qiao
Chao-Cheng Wu
Chein-I. Chang




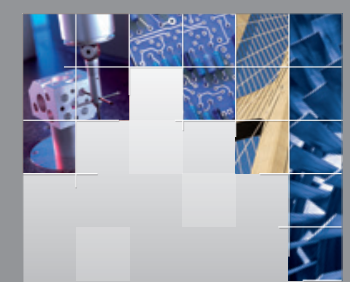

\section{Enfincering}
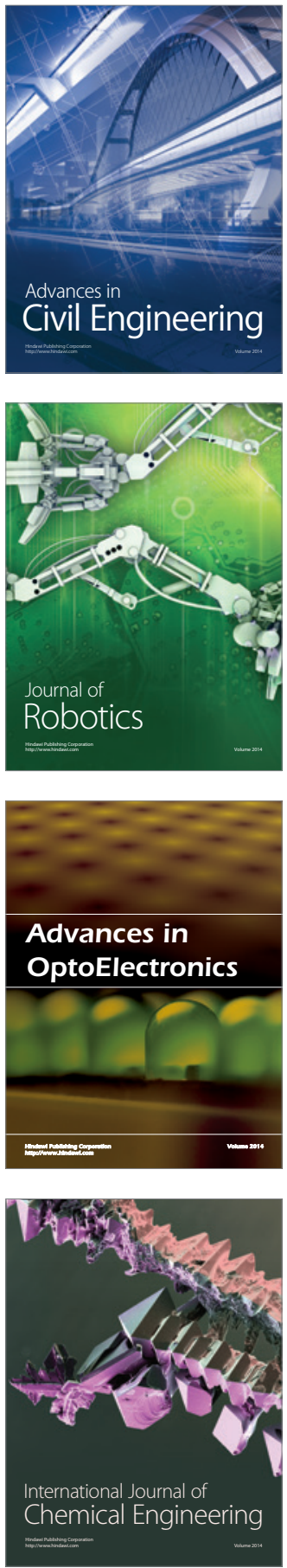

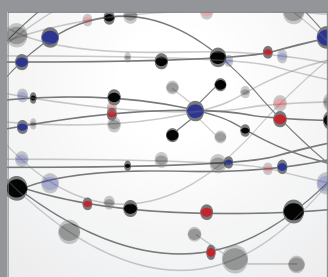

The Scientific World Journal

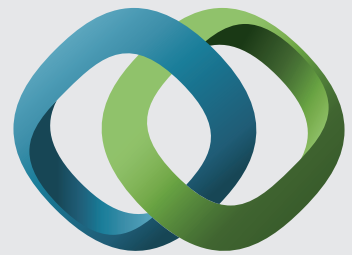

\section{Hindawi}

Submit your manuscripts at

http://www.hindawi.com
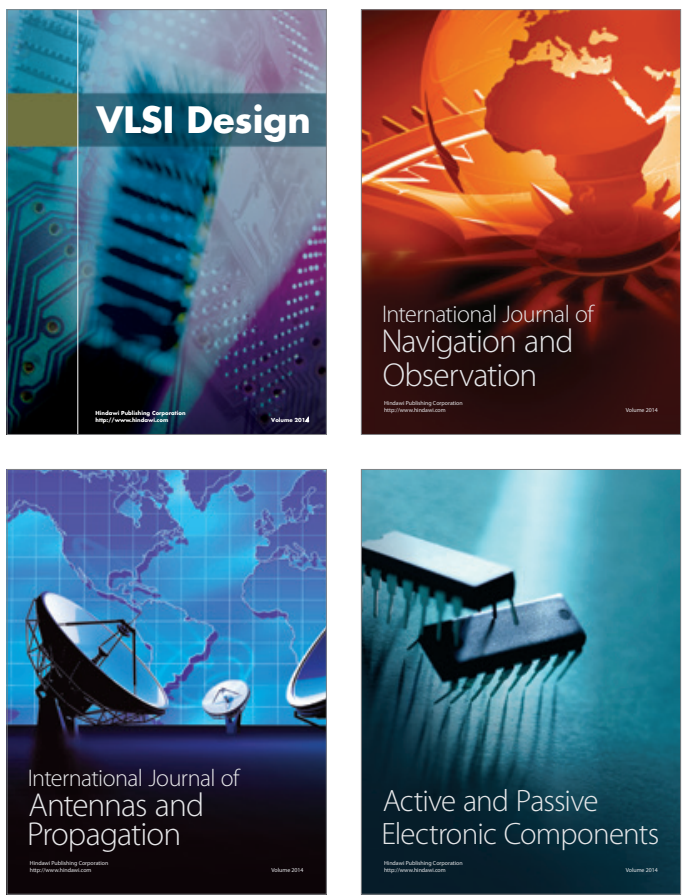
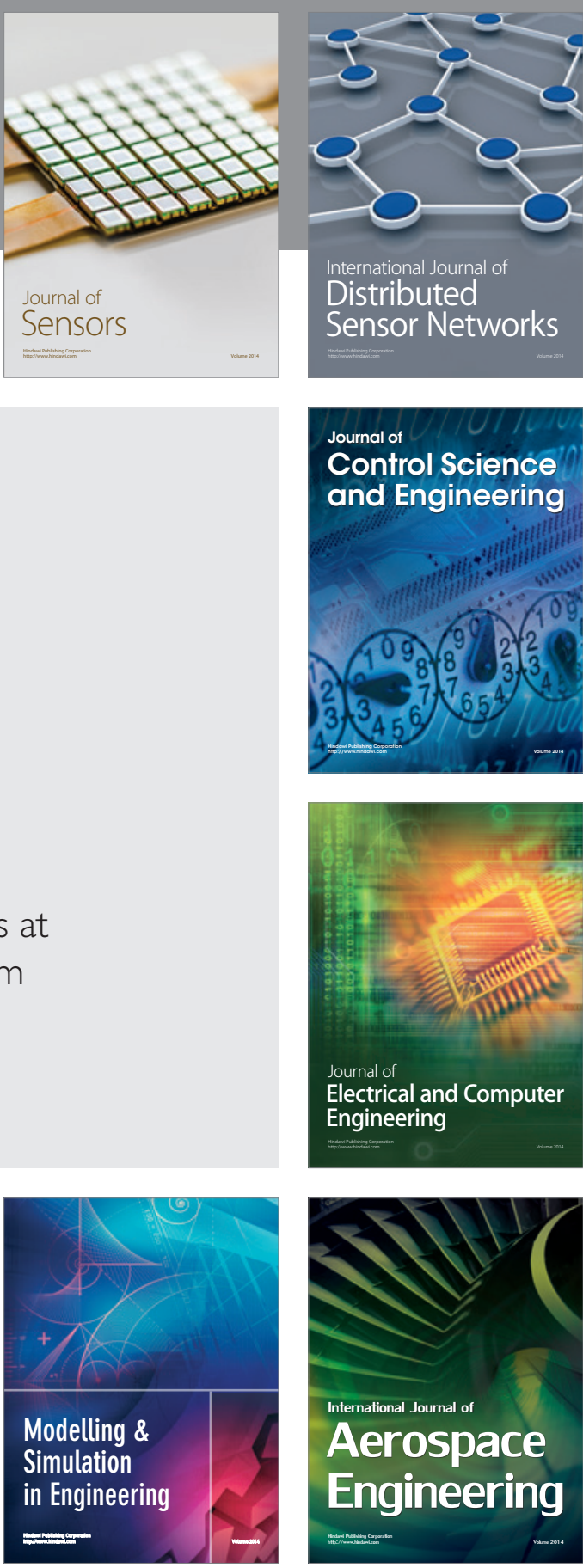

International Journal of

Distributed

Sensor Networks

Journal of

Control Science

and Engineering
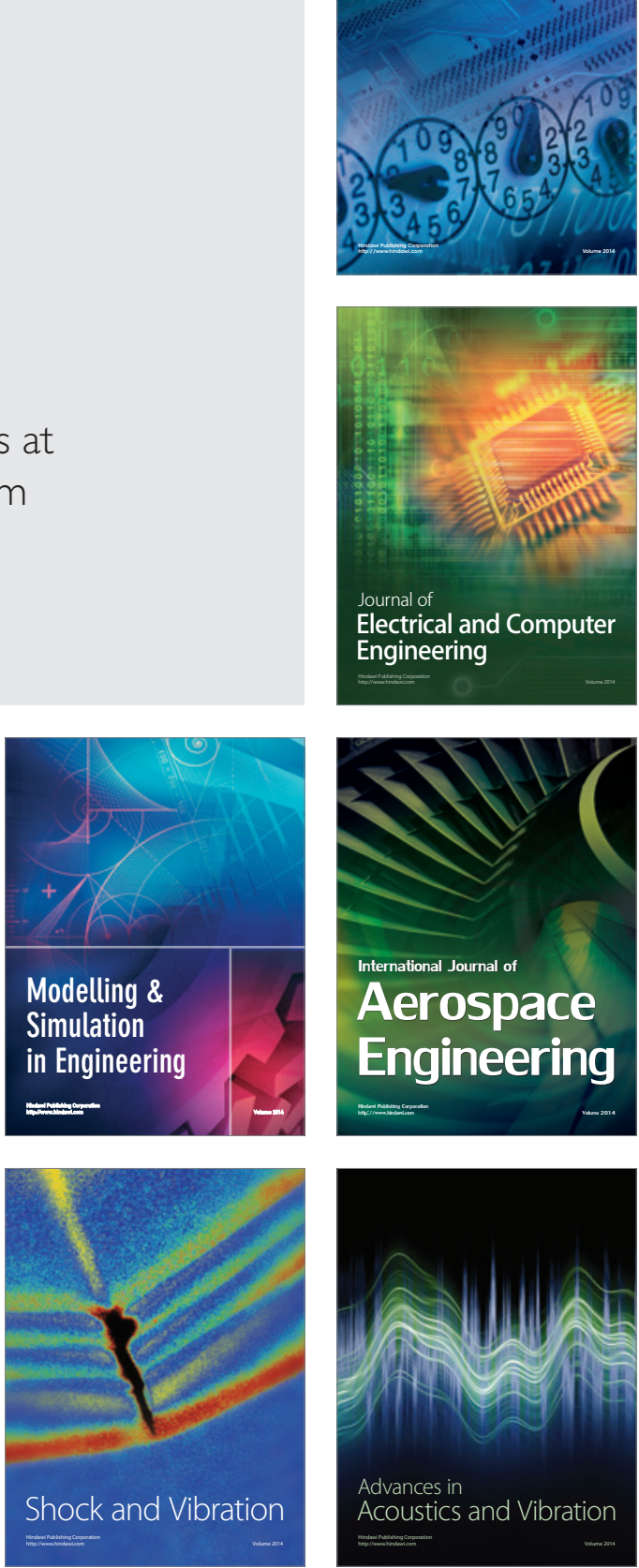